Research Article

\title{
Prevalence and Genotype Distribution of Giardia duodenalis in Rabbits in Shandong Province, Eastern China
}

\author{
Tao-Shan Li, ${ }^{1,2}$ Yang Zou $\mathbb{D}^{2},{ }^{2}$ Jun-Jie Peng, ${ }^{2}$ Li-Qun Wang, ${ }^{2}$ Hai-Sheng Zhang, ${ }^{2}$ Wei Cong, ${ }^{3}$ \\ Xing-Quan Zhu $\mathbb{1}^{2,4}$ and Xiao-Lin Sun $\mathbb{1}^{1}$ \\ ${ }^{1}$ College of Veterinary Medicine, Gansu Agricultural University, Lanzhou, Gansu 730070, China \\ ${ }^{2}$ State Key Laboratory of Veterinary Etiological Biology, Key Laboratory of Veterinary Parasitology of Gansu Province, \\ Lanzhou Veterinary Research Institute, Chinese Academy of Agricultural Sciences, Lanzhou, Gansu 730046, China \\ ${ }^{3}$ Marine College, Shandong University at Weihai, Weihai, Shandong 250100, China \\ ${ }^{4}$ Jiangsu Co-Innovation Center for the Prevention and Control of Important Animal Infectious Diseases and Zoonoses, \\ Yangzhou University College of Veterinary Medicine, Yangzhou, Jiangsu 225009, China
}

Correspondence should be addressed to Yang Zou; zouyangdr@163.com and Xiao-Lin Sun; sunxl@gsau.edu.cn

Received 19 August 2019; Revised 25 November 2019; Accepted 31 December 2019; Published 17 February 2020

Academic Editor: Kui Li

Copyright (c) 2020 Tao-Shan Li et al. This is an open access article distributed under the Creative Commons Attribution License, which permits unrestricted use, distribution, and reproduction in any medium, provided the original work is properly cited.

Giardia duodenalis is a zoonotic enteric parasite that can infect humans and a number of animal species including rabbits with a worldwide distribution. Infection with $G$. duodenalis can cause serious public health problems and significant economic losses to animal husbandry. So accurate understanding of the prevalence and genotype distribution of G. duodenalis in rabbits is necessary. In the present study, a total of $616 \mathrm{fecal}$ samples were collected from rabbits in Shandong province, eastern China, and examined in G. duodenalis prevalence and genotypes by nested PCR amplification of $\beta$-giardin (bg), glutamate dehydrogenase (gdh), and triosephosphate isomerase (tpi) gene loci of G. duodenalis. Sixty-nine (11.2\%) of the examined rabbit fecal samples were G. duodenalis-positive. Of them, the prevalence of G. duodenalis is 8.4\% (41/490) in Rizhao city and 22.2\% (28/126) in Weihai city. Breeds, region, and feeding modes were highly correlated with G. duodenalis infection in rabbits. Moreover, three genotypes (assemblages A, B, and E) were identified in rabbits at three gene loci, and the assemblage $\mathrm{E}$ was the dominant genotype, while the assemblage A was reported in rabbits in China for the first time. It is noticeable that two rabbits were found to be infected with two different G. duodenalis assemblages (assemblages A and E, assemblages B and E, respectively). These findings enrich the genotype distribution of G. duodenalis in rabbits and provide baseline data for preventing and controlling G. duodenalis infection in rabbits in eastern China.

\section{Introduction}

Giardia duodenalis (syn. Giardia intestinalis, Giardia lamblia) is a common gastrointestinal protozoon that causes enteric disease in a variety of animal species and humans [1-4]. More than 40 species of animals have been reported to be infected with $G$. duodenalis over the world $[2,5]$. Humans can be infected through ingesting water and food contaminated with $G$. duodenalis cysts [6]. The clinical symptoms of giardiasis are diarrhea, dehydration, abdominal pain, nausea, vomiting, and weight loss $[2,6,7]$. Giardiasis caused by $G$. duodenalis also has been recognized as an important zoonotic disease for both public and animal health $[2,7,8]$.

Eight assemblages have been identified in G. duodenalis, including assemblages A-H $[2,5]$. The structure of eight assemblages is similar, but the genotypes are distinct [8]. Among assemblages $\mathrm{A}-\mathrm{H}$, both of the assemblages $\mathrm{A}$ and $\mathrm{B}$ are usually identified in various mammals, including humans [9], while the remaining assemblages mainly occur in the relatively specific groups of animals $[10,11]$.

Rabbits are one of the most important economic animals in China, and the consumption of meat and fur accounts for a large part of China's economy [12]. However, rabbits are 
TABLE 1: Nest PCR primers and annealing temperatures used in this study.

\begin{tabular}{|c|c|c|c|c|c|}
\hline Gene & Primer & Sequence $\left(5^{\prime}-3^{\prime}\right)$ & Annealing temperature $\left({ }^{\circ} \mathrm{C}\right)$ & Length (bp) & Reference \\
\hline \multirow{4}{*}{ bg } & F1 & AAGCCCGACGACCTCACCCGCAGTGC & 55 & & {$[2]$} \\
\hline & $\mathrm{R} 1$ & GAGGCCGCCCTGGATCTTCGAGACGAC & & & \\
\hline & $\mathrm{F} 2$ & GAACGAACGAGATCGAGGTCCG & 55 & 511 & \\
\hline & $\mathrm{R} 2$ & CTCGACGAGCTTCGTGTT & & & \\
\hline \multirow{4}{*}{ gdh } & F1 & TTCCGTRTYCAGTACAACTC & 50 & & {$[2]$} \\
\hline & $\mathrm{R} 1$ & ACCTCGTTCTGRGTGGCGCA & & & \\
\hline & $\mathrm{F} 2$ & ATGACYGAGCTYCAGAGGCACGT & 65 & 530 & \\
\hline & $\mathrm{R} 2$ & GTGGCGCARGGCATGATGCA & & & \\
\hline \multirow{4}{*}{ tpi } & F1 & AAATIATGCCTGCTCGTCG & 55 & & [2] \\
\hline & $\mathrm{R} 1$ & CAAACCTTITCCGCAAACC & & & \\
\hline & $\mathrm{F} 2$ & CCCTTCATCGGIGGTAACTT & 55 & 530 & \\
\hline & $\mathrm{R} 2$ & GTGGCCACCACICCCGTGCC & & & \\
\hline
\end{tabular}

susceptible to many pathogens $[5,13,14]$, including $G$. duodenalis [15-20], which can cause significant economic losses to the rabbit breeding industry. There are limited reports about $G$. duodenalis prevalence in rabbits in China $[15,18-20]$; therefore, the objective of the present study was to investigate the prevalence and genotype distribution of G. duodenalis in rabbits in Shandong province, eastern China.

\section{Materials and Methods}

2.1. Collection of Fecal Samples. A total of 616 rabbit fecal samples (490 from Rizhao city, 126 from Weihai city) were collected from Shandong province, eastern China. The fecal samples were composed of 207 New Zealand white rabbits, 188 long-haired rabbits, and 221 Tolai hares. Each fresh fecal sample was collected with gloves and placed into a box with ice, then marked with the breed, age, and region, respectively, and transported to the laboratory. All of the fecal samples were stored at $-20^{\circ} \mathrm{C}$ for further DNA extraction.

2.2. Genomic DNA Extraction. Each rabbit fecal sample was washed with distilled water, uniformly stirred, and the residue was filtered through a 60-mesh sieve $(0.23 \times 0.23 \mathrm{~mm})$. The filtrate was centrifuged at $4000 \mathrm{~g}$ for $5 \mathrm{~min}$ in a centrifuge, the filtrate was discarded, and the precipitate was retained; then approximately $300 \mathrm{mg}$ of each precipitate of fecal sample was used to extract genomic DNA using the commercial E.Z.N.A.R ${ }^{\circledR}$ Stool DNA Kit (Omega Bio-Tek Inc., Norcross, GA, USA), following the manufacturer's recommendations. The extracted genomic DNA was stored at $-20^{\circ} \mathrm{C}$ for further PCR amplification.

2.3. Nested PCR Amplification. The prevalence and genotypes of $G$. duodenalis were examined by nested PCR amplification of the bg, gdh, and tpi gene loci as described previously $[2,15]$. The primers and corresponding annealing temperature are shown in Table 1. Positive and negative controls were included in each PCR reaction. The secondary PCR products were electrophoresed in 1\% (w/v) agarose gels containing ethidium bromide.
2.4. Sequence Analysis and Phylogeny. All of the positive secondary PCR products were sent to Tsingke Biotechnology Technology Company (Xi'an city, China) for two-directional sequencing. The obtained sequences were compared with reference sequences in GenBank database (http://www.ncbi. nlm.nih.gov/BLAST/) and edited using computer Clustal X 1.83 [21]. The phylogenetic tree was constructed using the Neighbor-Joining [NJ] analysis in MEGA7 (https://www. megasoftware.net/), the Kimura 2-parameter model was selected, and 1,000 bootstrap replicates were calculated.

2.5. Statistical Analysis. The relationship between the G. duodenalis prevalence in rabbits with different variables such as breed, region, age, and feeding mode was analyzed by the Chi-square $\left(\chi^{2}\right)$ test. The $95 \%$ confidence intervals (CIs) and odds ratios (ORs) were estimated by SPSS version 24.0 (SPSS Inc., Chicago, IL, USA). If the statistical result $P<0.05$, the difference was considered statistically significant.

\section{Results and Discussion}

3.1. The Prevalence of G. duodenalis in Rabbits. In the present study, 69 (11.2\%, 95\% CI: 8.71-13.69) of the examined 616 rabbit fecal samples were positive for $G$. duodenalis by nested PCR amplification of the bg, gdh, and tpi loci (Table 2). Among different breeds, the highest prevalence was $23.1 \%$ (95\% CI: 17.52-28.63) in Tolai hares, followed by $7.3 \%(95 \%$ CI: 3.72-10.78) in New Zealand white rabbits and 1.6\% (95\% CI: 0.19-3.39) in long-haired rabbits, and the difference was considered significant $\left(\chi^{2}=50.03, \mathrm{~d} f=2, P<0.01\right)$. Moreover, the $G$. duodenalis prevalence in rabbits from Rizhao city $(8.4 \%$, 95\% CI: 5.92-10.82) was significantly lower than that from Weihai city $(22.2 \%$, 95\% CI: 14.96-29.48) $\left(\chi^{2}=19.34, \mathrm{~d} f=1, P<0.01\right)$. Rabbits raised outdoors had a significantly higher $G$. duodenalis prevalence $(23.1 \%)$ than that raised indoors $(4.6 \%)\left(\chi^{2}=48.87, \mathrm{~d} f=1, P<0.01\right)$ (Table 2). Rabbits that are less than 6 months had a slightly lower G. duodenalis prevalence of 9.4\% (95\% CI: 5.93-12.77) than rabbits of more than 6 months old $(12.7 \%, 95 \% \mathrm{CI}$ : 9.17-16.27) (Table 2). The G. duodenalis prevalence in male rabbits (11.5\%) (95\% CI: 7.70-15.33) was slightly higher $\left(\chi^{2}=0.05, \mathrm{~d} f=1, P=0.82\right)$ than that in female rabbits (11.0\%) (95\% CI: 7.67-14.22) (Table 2). 
TABle 2: Prevalence and risk factors of Giardia duodenalis infection in rabbits.

\begin{tabular}{|c|c|c|c|c|c|c|}
\hline Factor & Category & No. tested & No. positive & $\%(95 \% \mathrm{CI})$ & $P$ value & OR $(95 \% \mathrm{CI})$ \\
\hline \multirow{3}{*}{ Breeds } & Long-haired rabbit & 188 & 3 & $1.6(0.19-3.39)$ & \multirow[t]{3}{*}{0.01} & Reference \\
\hline & New Zealand white rabbit & 207 & 15 & $7.3(3.72-10.78)$ & & $4.82(1.37-16.92)$ \\
\hline & Tolai hare & 221 & 51 & $23.1(17.52-28.63)$ & & $18.50(5.67-60.38)$ \\
\hline \multirow{2}{*}{ Region } & Rizhao & 490 & 41 & $8.4(5.92-10.82)$ & \multirow[t]{2}{*}{0.01} & Reference \\
\hline & Weihai & 126 & 28 & $22.2(14.96-29.48)$ & & $3.13(1.85-5.30)$ \\
\hline \multirow{2}{*}{ Feeding } & Indoors & 395 & 18 & $4.6(2.50-6.62)$ & \multirow[t]{2}{*}{0.01} & Reference \\
\hline & Outdoors & 221 & 51 & $23.1(17.50-28.64)$ & & $6.28(3.56-11.08)$ \\
\hline \multirow{2}{*}{ Age } & Young ( $<6$ months) & 278 & 26 & $9.4(5.93-12.77)$ & \multirow[t]{2}{*}{0.19} & Reference \\
\hline & Adult ( $\geq 6$ months) & 338 & 43 & $12.7(9.17-16.27)$ & & $1.41(0.84-2.37)$ \\
\hline \multirow{2}{*}{ Gender } & Female & 347 & 38 & $11.0(7.67-14.22)$ & \multirow[t]{2}{*}{0.82} & Reference \\
\hline & Male & 269 & 31 & $11.5(7.70-15.33)$ & & $1.06(0.64-1.75)$ \\
\hline Total & & 616 & 69 & $11.2(8.71-13.69)$ & & \\
\hline
\end{tabular}

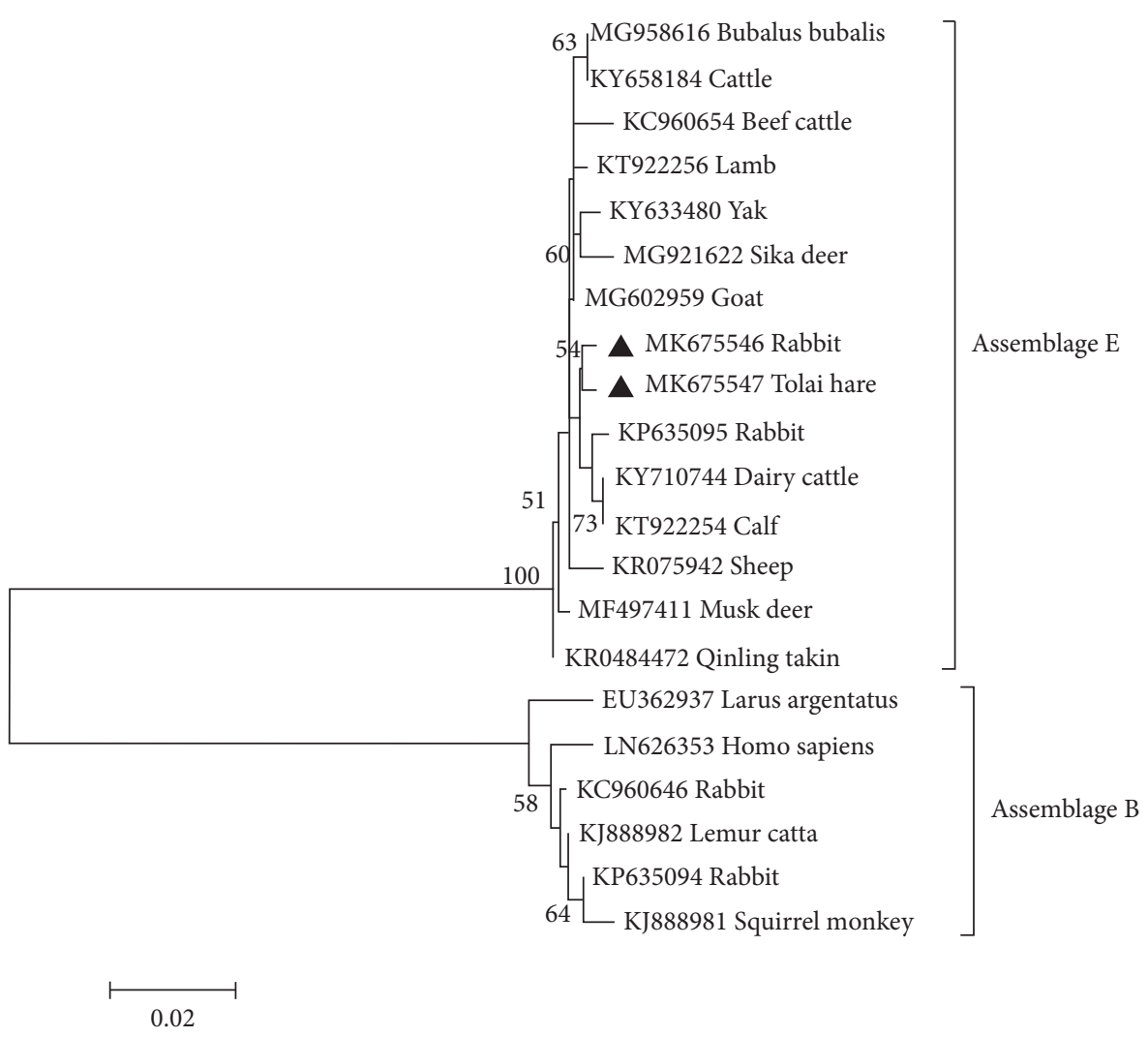

Figure 1: The phylogenetic relationships among G. duodenalis isolates. The numbers on the branches represent percent bootstrapping values from 1000 replicates, with values of more than 50\% shown in the tree. The genotypes of G. duodenalis which were identified at gdh gene locus in the present study are marked by filled triangles.

In the present study, the overall G. duodenalis prevalence in rabbits was $11.2 \%(69 / 616)$, which was higher than that in rabbits in Henan province $(8.4 \%, 80 / 955)$ [15], Jilin province and Liaoning province $(9.86 \%, 42 / 426)$ [18], Heilongjiang province $(7.41 \%, 28 / 378)$ [20], Xinjiang province $(1.9 \%, 6 /$ 321) [19] in China, and in Europe $(7.6 \%, 40 / 528)$ [16] and Melbourne, Australia $(1.03 \%, 1 / 97)$ [22], but lower than that in rabbits in Ecuador $(20.0 \%, 4 / 20)$ [17]. The different $G$. duodenalis prevalence in rabbits may be caused by many factors such as geographical ecological environment, detection methods, sample size, and individual health status [23].
3.2. Molecular Characterization of G. duodenalis Isolates. Two G. duodenalis assemblages (assemblages B and E) have been reported in rabbits in China [15, 18-20]. In the present study, 616 rabbit fecal samples were used to identify G. duodenalis genotypes by nested PCR targeting the bg, gdh, and tpi genes. The results showed that 53 bg-positive samples and 39 gdh-positive samples were identified as assemblage E; two $(2.9 \%, 2 / 69)$ tpi-positive samples and one $(1.5 \%, 1 / 69)$ tpi-positive sample were identified as assemblage $\mathrm{B}$ and assemblage A, respectively. Interestingly, the assemblage $\mathrm{E}(98.6 \%, 68 / 69)$ was the dominant genotype in 


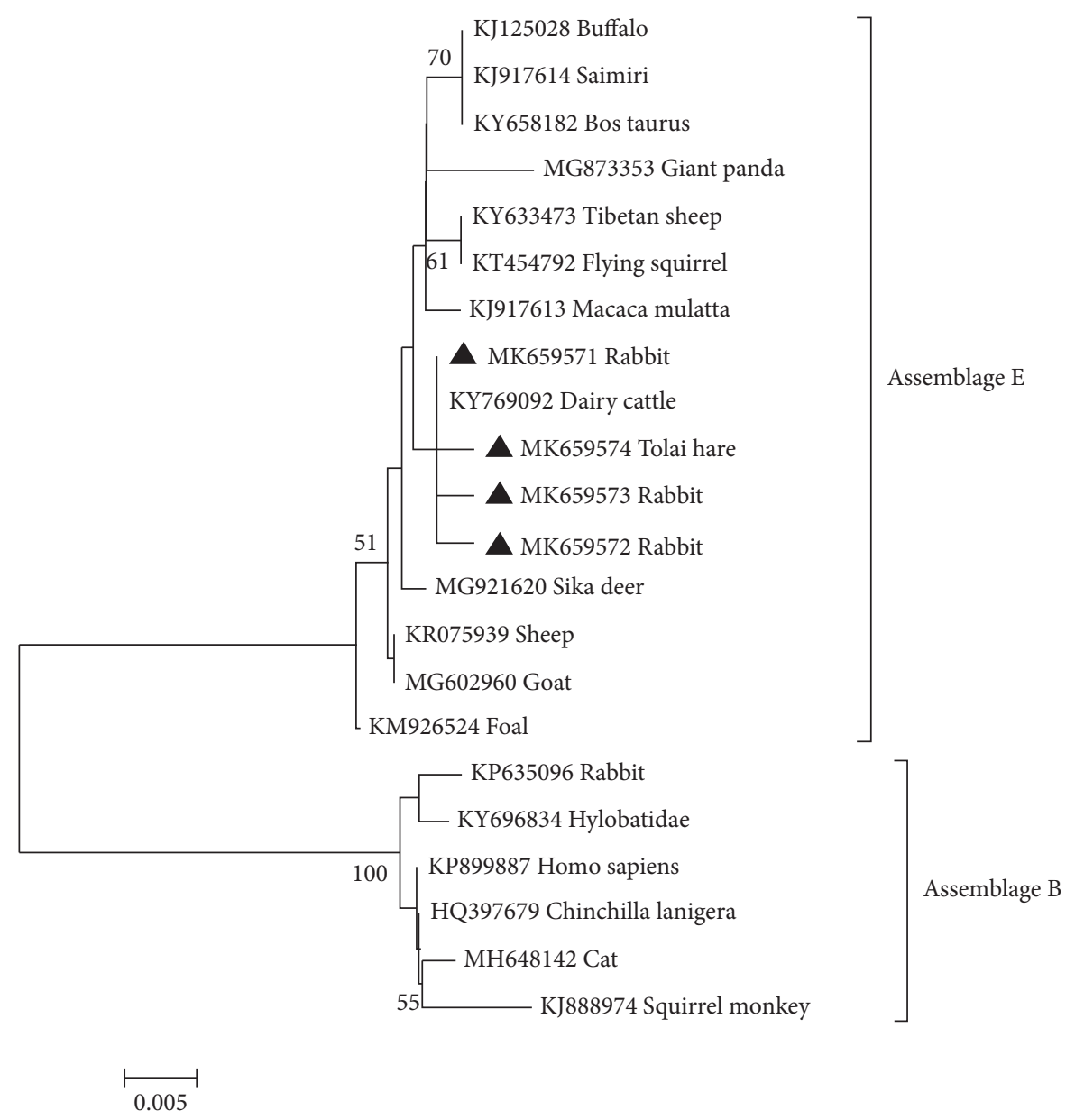

FIGURE 2: The phylogenetic relationships among G. duodenalis isolates inferred by a Neighbor-Joining (NJ) algorithm using a Kimura twoparameter analysis (1000 replicates) based on the bg gene sequences. The genotypes of $G$. duodenalis which were identified at bg gene locus in the present study are marked by filled triangles.

rabbits in the present study, which was different from the previous studies $[18,19]$. Moreover, two mixed $G$. duodenalis assemblages (assemblages $\mathrm{A}$ and $\mathrm{E}$, assemblages $\mathrm{B}$ and E) were identified in rabbits, and the assemblage A was firstly detected in rabbits in China in the present study. The G. duodenalis assemblage $\mathrm{A}$ was also reported in humans $[9,22]$. This finding suggests that the rabbits may be a potential source of human infection with $G$. duodenalis.

\subsection{Phylogenetic Analysis of G. duodenalis Isolates in Rabbits.} To further elucidate the genetic relationship of $G$. duodenalis assemblages in rabbits, we aligned the obtained sequences with reference sequences in GenBank by Clustal X 1.83, which were used for phylogenetic analyses (Figures 1 and 2). The phylogenetic analyses showed that the G. duodenalis assemblage $\mathrm{E}$ in rabbits and dairy cattle was distributed on one branch, representing a closer genetic relationship (Figure 2). These findings indicated a possibility of spreading G. duodenalis between rabbits and dairy cattle. Moreover, the single nucleotide polymorphisms (SNPs) existed in bg sequences and gdh sequences in this study (Table 3) by comparing the obtained $G$. duodenalis sequences in the present study with corresponding sequences in the GenBank
TABLE 3: Variations in bg and gdh gene loci among the subtypes of Giardia duodenalis assemblage E in rabbits.

\begin{tabular}{lcccccc}
\hline Isolate & Subtype (no.) & \multicolumn{6}{c}{ Nucleotide at position } & GenBank ID \\
\hline & & 83 & 201 & 429 & 471 & \\
bg & Ref. sequence & A & A & C & C & KU886048 \\
JT-32 & E1 $(n=1)$ & - & - & - & T & MK659571 \\
JT-42 & E2 $(n=1)$ & - & G & - & - & MK659572 \\
JT-334 & E3 $(n=1)$ & G & - & - & - & MK659573 \\
YT-10 & E4 $(n=1)$ & - & - & G & - & MK659574 \\
& & 24 & 399 & & & \\
gdh & Ref. sequence & A & A & & & KF843962 \\
JT-37 & E1 $(n=1)$ & - & G & & & MK675546 \\
YT-189 & E2 $(n=1)$ & C & & & & MK675547 \\
\hline
\end{tabular}

database. These findings indicated that various $G$. duodenalis types are distributed in rabbits. These findings enriched the genetic diversity of $G$. duodenalis in rabbits and other animals.

\section{Conclusions}

The present study revealed a higher (11.2\%) G. duodenalis prevalence in rabbits in Shandong province, eastern China. Different feeding methods, breeds, and regions were highly 
correlated with $G$. duodenalis prevalence in rabbits $(P<0.05)$. Three G. duodenalis assemblages (assemblage A, assemblage $\mathrm{B}$, and assemblage $\mathrm{E}$ ) were identified in rabbits, assemblage $\mathrm{A}$ was reported in rabbits in China for the first time, while assemblage $\mathrm{E}$ was the dominant assemblage. These findings not only enriched the genotype distribution of G. duodenalis in rabbits but also have an implication for better control of $G$. duodenalis in rabbits. Further studies are necessary to examine the infection status and genotype distribution of $G$. duodenalis in rabbits in other areas of the country.

\section{Data Availability}

The G. duodenalis prevalence data used to support the findings of this study are included in the article.

\section{Ethical Approval}

All rabbits were handled in strict accordance with good animal practice according to the Animal Ethics Procedures and Guidelines of the People's Republic of China, and the study was approved by the Animal Administration and Ethics Committee of Lanzhou Veterinary Research Institute, Chinese Academy of Agricultural Sciences.

\section{Conflicts of Interest}

The authors declare that they have no conflicts of interest.

\section{Acknowledgments}

This project was supported by the Agricultural Science and Technology Innovation Program (ASTIP) (Grant no. CAASASTIP-2016-LVRI-03) and the Elite Program of the Chinese Academy of Agricultural Sciences.

\section{References}

[1] Y. Jin, J. Fei, J. Cai et al., "Multilocus genotyping of Giardia duodenalis in Tibetan sheep and yaks in Qinghai, China," Veterinary Parasitology, vol. 247, pp. 70-76, 2017.

[2] Y. Feng and L. Xiao, "Zoonotic potential and molecular epidemiology of Giardia species and giardiasis," Clinical Microbiology Reviews, vol. 24, no. 1, pp. 110-140, 2011.

[3] G. Certad, E. Viscogliosi, M. Chabé, and S. M. Cacciò, "Pathogenic mechanisms of Cryptosporidium and Giardia," Trends in Parasitology, vol. 33, no. 7, pp. 561-576, 2017.

[4] Y.-L. Yin, H.-J. Zhang, Y.-J. Yuan et al., "Prevalence and multi-locus genotyping of Giardia duodenalis from goats in Shaanxi province, northwestern China," Acta Tropica, vol. 182, pp. 202-206, 2018.

[5] U. Ryan, N. Hijjawi, Y. Feng, and L. Xiao, "Giardia: an underreported foodborne parasite," International Journal for Parasitology, vol. 49, no. 1, pp. 1-11, 2019.

[6] M. C. Halliez and A. G. Buret, "Extra-intestinal and long term consequences of Giardia duodenalis infections," World Journal of Gastroenterology, vol. 19, no. 47, pp. 8974-8985, 2013.

[7] S. A. Squire and U. Ryan, "Cryptosporidium and Giardia in Africa: current and future challenges," Parasites and Vectors, vol. 10, no. 1, p. 195, 2017.
[8] E. Einarsson, S. Ma'ayeh, and S. G. Svärd, "An up-date on Giardia and giardiasis," Current Opinion in Microbiology, vol. 34, pp. 47-52, 2016.

[9] Y. Wang, N. Li, Y. Guo et al., "Persistent occurrence of Cryptosporidium hominis and Giardia duodenalis subtypes in a Welfare Institute," Frontiers in Microbiology, vol. 9, p. 2830, 2018.

[10] X. Wang, M. Cai, W. Jiang et al., "High genetic diversity of Giardia duodenalis assemblage E in pre-weaned dairy calves in Shanghai, China, revealed by multilocus genotyping," Parasitology Research, vol. 116, no. 8, pp. 2101-2110, 2017.

[11] X. X. Zhang, W. B. Zheng, J. G. Ma et al., "Occurrence and multilocus genotyping of Giardia intestinalis assemblage C and $\mathrm{D}$ in farmed raccoon dogs, Nyctereutes procyonoides, in China," Parasites and Vectors, vol. 9, no. 1, p. 471, 2016.

[12] S. Li, W. Zeng, R. Li et al., "Rabbit meat production and processing in China," Meat Science, vol. 145, pp. 320-328, 2018.

[13] Z. Yang, W. Zhao, Y. Shen et al., "Subtyping of Cryptosporidium cuniculus and genotyping of Enterocytozoon bieneusi in rabbits in two farms in Heilongjiang Province, China," Parasite, vol. 23, p. 52, 2016.

[14] X. Xie, J. Bil, E. Shantz, J. Hammermueller, E. Nagy, and P. V. Turner, "Prevalence of lapine rotavirus, astrovirus, and hepatitis E virus in Canadian domestic rabbit populations," Veterinary Microbiology, vol. 208, pp. 146-149, 2017.

[15] M. Qi, J. Xi, J. Li, H. Wang, C. Ning, and L. Zhang, "Prevalence of Zoonotic Giardia duodenalis assemblage B and first identification of assemblage $\mathrm{E}$ in rabbit fecal samples isolates from central China," Journal of Eukaryotic Microbiology, vol. 62, no. 6, pp. 810-814, 2015.

[16] N. Pantchev, A. Broglia, B. Paoletti et al., "Occurrence and molecular typing of Giardia isolates in pet rabbits, chinchillas, guinea pigs and ferrets collected in Europe during 2006-2012," Veterinary Record, vol. 175, no. 1, p. 18, 2014.

[17] K. Vasco, J. P. Graham, and G. Trueba, "Detection of zoonotic enteropathogens in children and domestic animals in a semirural community in Ecuador," Applied and Environmental Microbiology, vol. 82, no. 14, pp. 4218-4224, 2016.

[18] J. Jiang, J.-G. Ma, N.-Z. Zhang et al., "Prevalence and risk factors of Giardia duodenalis in domestic rabbbits (Oryctolagus cuniculus) in Jilin and Liaoning province, northeastern China," Journal of Infection and Public Health, vol. 11, no. 5, pp. 723-726, 2018.

[19] X. Zhang, M. Qi, B. Jing et al., "Molecular characterization of Cryptosporidium spp., Giardia duodenalis, and Enterocytozoon bieneusi in rabbits in Xinjiang, China," Journal of Eukaryotic Microbiology, vol. 65, no. 6, pp. 854-859, 2018.

[20] W. Zhang, Y. Shen, R. Wang et al., "Cryptosporidium cuniculus and Giardia duodenalis in rabbits: genetic diversity and possible zoonotic transmission," PLoS One, vol. 7, no. 2, Article ID e31262, 2012.

[21] J. D. Thompson, T. J. Gibson, F. Plewniak et al., "The CLUSTAL_X windows interface: flexible strategies for multiple sequence alignment aided by quality analysis tools," Nucleic Acids Research, vol. 25, no. 24, pp. 4876-4882, 1997.

[22] A. V. Koehler, S. R. Haydon, A. R. Jex, and R. B. Gasser, "Cryptosporidium and Giardia taxa in faecal samples from animals in catchments supplying the city of Melbourne with drinking water (2011 to 2015)," Parasites and Vectors, vol. 9, no. 1, p. 315, 2016.

[23] L. Deng, W. Li, Z. Zhong et al., "Prevalence and molecular characterization of Giardia intestinalis in racehorses from the Sichuan province of southwestern China," PLoS One, vol. 12, no. 12, Article ID e0189728, 2017. 